\title{
An Analytic Method for \\ Two-Dimensional Wall Motion and \\ Product Isentrope from the \\ Detonation Cylinder Test
}

\author{
Scott I. Jackson \\ Shock and Detonation Physics Group \\ Los Alamos National Laboratory, Los Alamos, NM 87545 USA
}

\begin{abstract}
The cylinder test provides a measurement of detonation product's ability to perform work on adjacent material. Historically, direct numerical simulation has been required to derive the product energy content and isentrope from experiments of cylinder expansion driven by detonation products. One-dimensional analytic methods have not been able to accurately recover these parameters when the cylinder motion is compressible, exhibiting shocks. For incompressible cylinder motion, analytic one-dimensional approximations more accurately recover the isentrope, but still only approximate the two-dimensional cylinder motion and energy. This work provides a fully two-dimensional model that recovers the exact outer cylinder shape from experimental measurements. The inner cylinder shape and product isentrope are also exactly recovered in the limit of incompressible case motion. An alternate methodology also approximates the inner case shape and isentrope for compressible case motion, effectively allowing accurate isentrope determination for any cylinder
\end{abstract}


test. The isentrope derived from a PBX 9502 cylinder test with compressible motion is shown to agree well with a reference isentrope. The errors associated with the one-dimensional flow assumption are also quantified. The incompressible case model can be used to estimate case shape and velocity from a given isentrope, providing a maximum fragment velocity.

Key words: Detonation, Cylinder Test, Equation of State

Topic: Detonations, Explosions, and Supersonic Combustion

\section{Nomenclature}

$A$ - product flow geometry coefficient

$a_{0}-$ initial wall acceleration

$D$ - detonation velocity

$e-$ specific energy

$F_{\mathrm{b}}$ - axial pressure force

$P$ - product pressure

$P_{\mathrm{r}}-$ Rayleigh line pressure

$R$ - initial radius

$r$ - radius

$t$ - time

$u$ - axial velocity

$V$ - total velocity

$v_{\mathrm{o}}$ - radial outer wall velocity

$v_{\text {expt }}$ - experimental $v_{o}$

$v_{\infty}-$ terminal $v_{o}$

$x$ - axial coordinate

$\eta$ - geometry toggle

$d \ell$ - differential wall length element

$\Lambda$ - linear JWL coefficient 
$\lambda$ - nonlinear JWL coefficient

$\rho$ - density

$\theta$ - angle

$\nu$ - specific volume, $1 / \rho$

$\omega$ - exponential fit parameter

0 - initial state

CJ - Chapman-Jouguet state

c - compressible wall model

e - initial explosive state

i - inner wall state

ic - incompressible wall model

m - center-of-mass state

o - outer wall state

p - product state

w - wall state

\section{Introduction}

The detonation cylinder test is a standard performance experiment used to evaluate the capability of a condensed explosive to accelerate confining material [1-4]. The test configuration consists of an inert confiner tube tightly encasing a high explosive rod. Detonation is initiated at one end and travels along the rod length. High-pressure products accelerate the confiner outwards (as shown in Fig. 1) and the motion history is recorded with a streak camera or Doppler-velocity-probe diagnostic. The confiner rapidly accelerates at first and then approaches a terminal velocity at later times, as most of the energy is extracted from the expanded product gas.

Traditionally, the velocity at specific radii are used as coarse performance metrics, while the full velocity profile is used to empirically calibrate equation-of-state (EOS) parameters for the product gas. The latter process is computationally intensive: Cylinder motion is computed via direct numerical 
simulation from an initial EOS guess. If the calculated motion does not match the experiment, the EOS is modified and cylinder motion is recomputed. This process iterates until the predicted motion acceptably approximates experiment. Typically, the resulting EOS data is presented in a standard JWL (Jones-Wilkins-Lee) isentrope form [5] appearing as $P=\Lambda_{1} e^{-\lambda_{1} \frac{\nu}{\nu_{0}}}+\Lambda_{2} e^{-\lambda_{2} \frac{\nu}{\nu_{0}}}+\Lambda_{3} \frac{\nu}{\nu_{0}}-\left(\lambda_{3}+1\right)$ where all $\Lambda$ and $\lambda$ values $^{1}$ are fitted coefficients. This approach can be sensitive to the code quality, mesh resolution, and other computational input parameters (reaction model, confiner EOS, etc.). This iterative hydrocode procedure is quite time intensive and not accessible to many researchers, indicating a need for an analytic method to determine the cylinder case motion and product isentrope from experimental measurements. In a reference frame attached to the detonation front, the cylinder motion is steady and thus accessible to analytic efforts. Taylor [6] first recognized the potential of the experimental geometry before any data existed. He noted that the cylinder test geometry did not subject the product gas to any shocks, allowing for isentropic expansion. He presented an analytic, one-dimensional (1-D) methodology to relate the radial cylinder motion to the product isentrope by neglecting radial product flow, axial cylinder motion, case compressibility and case shear strength.

Hill [7] utilized Taylor's [6] 1-D concepts to analytically determine the isentrope for (HMX-based) PBX 9501 detonation products from experimental cylinder motion. While compressibility artifacts, in the form of ringing, were present in the case motion, Hill used an analytic fitting form to smooth the data. The resulting isentrope predicted lower pressures for equivalent specific product volume $\nu_{\mathrm{p}}$ as compared to a (hydrocode-fitted) reference JWL isentrope. Hill [7] assumed that predicted pressures were correct, that the model failed to accurately predict $\nu_{\mathrm{p}}$, and proposed an empirical correction. Davis and Hill [8] later measured product isentropes for Ammonium-Nitrate-Fuel-Oil (ANFO) cylinder tests, where the case compressibility was less significant, and saw good agreement with calculation. The author is not aware of any other analytic efforts devoted to the cylinder test. This may be because Taylor's approach, while elegant in nature, underpredicts cylinder expansion and velocity. The error induced increases with the ratio of the radial case velocity $v_{\mathrm{o}}$ to the detonation velocity $D$ and is significant during the late stages of expansion.

$\overline{1}$ Unconventional JWL parameter symbols are used to avoid conflict with those in the following analysis. 
This work improves upon prior efforts $[6,7]$ and presents an analytic model to deduce the twodimensional (2-D) cylinder motion from a 1-D measurement of case expansion. The resulting motion is then used to infer the isentrope associated with the driving product gases. The 2-D model for the outer case shape is exact for any measured motion, while prediction of the inner case shape and product isentrope are only exact in the limit of an incompressible confiner with conical product flow. This method can also be reversed to predict the 2-D cylinder motion resulting from a specified EOS. An approximate analytic correction for compressible case motion, via shock impedance matching theory, is also presented. An example reduction of experimental cylinder data with compressible wall motion is compared to a reference JWL. The incompressible approach is shown to deviate in similar fashion to that in Hill [7] during compressible wall motion, while the compressible model agrees well with the JWL isentrope. When wall compressibility is no longer significant, the incompressible approach recovers the reference JWL. The results thus provide an analytic methodology to determine product isentropes from any cylinder test data and are easily adaptable to 2-D slab geometries as well.

\section{The Analytic Method}

Consider detonation propagation through an explosive rod confined by an inert tube. The explosive has initial density $\rho_{\mathrm{e}}$, initial radius $R_{\mathrm{i}}$ and detonates at a constant velocity $D$. The tube wall has initial density $\rho_{\mathrm{w}}$, inner radius $R_{\mathrm{i}}$, and outer radius $R_{\mathrm{o}}$. The detonation products are at pressure $P(x)$, assumed to be sufficiently large to neglect both the shear strength of the confiner ${ }^{2}$ and the external confiner pressure, $P_{\mathrm{e}}$. The explosive radius and confiner acceleration are assumed sufficiently large that diffusive heat transfer from the products to the confiner is not significant.

$\overline{2}$ For example, shear strength of annealed copper is below $0.2 \mathrm{GPa}$, while $P(x)$ for PBX 9502 will range from $30 \rightarrow 1 \mathrm{GPa}$ as the case expands from $R_{\mathrm{o}} \rightarrow 3 R_{\mathrm{o}}$. 


\subsection{Two-Dimensional Confiner Motion}

In a detonation-attached cylindrical coordinate system, $P(x)$ accelerates the confiner in a direction normal to the inner wall angle $\theta_{\mathrm{i}}(x)$. This acceleration causes the wall inner radius $r_{\mathrm{i}}(x)$ and outer radius $r_{\mathrm{o}}(x)$ to both increase with distance from the detonation, fixed at $x=0$, as shown in Fig. 1. As a result, the wall adopts a profile with positive slope for $x>0$ and the outer radius contains $\hat{x}^{-}$ and $\hat{r}$-velocity components $u_{\mathrm{o}}(x)$ and $v_{\mathrm{o}}(x)$, respectively, with $u_{\mathrm{o}}$ negative in sign.

Modern experimental methods measure wall motion with a Doppler velocimetry probe that is mounted in an Eulerian reference frame normal to the initial outer wall location and that records $v_{\mathrm{o}}(t)$. Thus, the following model specifies all variables via $v_{\mathrm{o}}(t)$, with first outer wall motion detected at $t=0$. Parameter $D$ is measured with time-of-arrival pins. The distance between the Eulerian probe and the detonation, $x=D t+\int u_{\mathrm{o}} d t$, allows transformation between $t$ and $x$ at the probe position.

Figure 2 illustrates that the outer wall angle $\theta_{\mathrm{o}}$ can be represented by both its instantaneous velocity vector, $\tan \theta_{\mathrm{o}}=-u_{\mathrm{o}} / v_{\mathrm{o}}$, and the motion of a differential wall element during time $d t$ such that, $\tan \theta_{\mathrm{o}}=\frac{d r_{\mathrm{o}} / d t}{d x / d t}=v_{\mathrm{o}} /\left(D+u_{\mathrm{o}}\right)$. Elimination of $\theta_{\mathrm{o}}$ yields the explicit solution,

$$
u_{\mathrm{o}}=\frac{-D+\left(D^{2}-4 v_{\mathrm{o}}^{2}\right)^{1 / 2}}{2}
$$

With $u_{\mathrm{o}}(t)$ known, the differential increase in horizontal probe position relative to the detonation is

$$
\frac{d x}{d t}=D+\eta u_{\mathrm{o}}
$$

and the differential increase in wall radius at the probe position is

$$
\frac{d r_{\mathrm{o}}}{d t}=v_{\mathrm{o}}-\eta u_{\mathrm{o}} \tan \theta_{\mathrm{o}}=v_{\mathrm{o}}+\eta \frac{u_{\mathrm{o}}^{2}}{v_{\mathrm{o}}}
$$

Parameter $\eta$ can be set to 0 or 1 for 1-D or 2-D analysis, respectively. The first component of Eq. (3) represents the actual outward motion of the wall. The second accounts for the radial increase from axial $(\hat{x})$ motion of the ramped wall at the Eulerian probe position. ${ }^{3}$ Numerical integration of

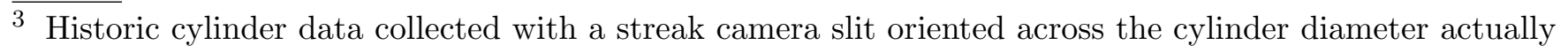


Eqs. (2) and (3) is straightforward with initial conditions $x(0)=D$ and $r_{\mathrm{o}}(0)=R_{\mathrm{o}}$. Indefinite integration may also be possible for some analytic representations of $v_{\mathrm{o}}(t)$.

With $r_{\mathrm{o}}(t)$ known, all other cylinder parameters are determined algebraically by assuming that the wall mass/length remains constant, yielding an inner radius of

$$
r_{\mathrm{i}}=\left[r_{\mathrm{o}}^{2}-\frac{\nu_{\mathrm{w}}}{\nu_{\mathrm{w}, 0}}\left(R_{\mathrm{o}}^{2}-R_{\mathrm{i}}^{2}\right)\right]^{1 / 2},
$$

where $\nu_{\mathrm{w}} / \nu_{\mathrm{w}, 0}=f(P)$ is the degree of volumetric confiner compression, which is unity for incompressible case motion. Case center-of-mass is

$$
r_{\mathrm{m}}=\left(\frac{r_{\mathrm{o}}^{2}+r_{\mathrm{i}}^{2}}{2}\right)^{1 / 2}=\left[r_{\mathrm{o}}^{2}-\frac{\nu_{\mathrm{w}}}{\nu_{\mathrm{w}, 0}} \frac{\left(R_{\mathrm{o}}^{2}-R_{\mathrm{i}}^{2}\right)}{2}\right]^{1 / 2}
$$

and the inner wall angle is

$$
\theta_{\mathrm{i}}=\frac{d r_{\mathrm{i}}}{d x}=\arctan \left[\frac{r_{\mathrm{o}}}{r_{\mathrm{i}}} \frac{\left(d r_{\mathrm{o}} / d t\right)}{(d x / d t)}\right] .
$$

Thus, Eqs. (1)-(6) allow reconstruction of the 2-D, incompressible confiner motion with knowledge of only $v_{\mathrm{o}}(t)$ and $D$. Equations (1)-(3) are exact for any type confiner motion, while Eqs. (4)-(6) require knowledge of $\nu_{\mathrm{w}} / \nu_{\mathrm{w}, 0}$ (and thus $P$ ) to remain exact during compressible case motion.

\subsection{Pressure for Incompressible Case Motion}

The product gas pressure $P$ acts on the inner wall area to accelerate it in a direction normal to $\theta_{\mathrm{i}}$. Specifically, the pressure force is $2 \pi r_{\mathrm{i}} P d \ell$ and the wall mass is $\pi \rho_{\mathrm{w}}\left(R_{\mathrm{o}}^{2}-R_{\mathrm{i}}^{2}\right) d \ell$, where $d \ell$ is a differential length wall element. The acceleration component detected by the velocimetry probe in the radial direction is $d v_{\mathrm{o}} / d t$. Application of Newton's second law to a differential wall element thus yields

$$
P=\frac{\rho_{\mathrm{w}}}{2} \frac{\left(R_{\mathrm{o}}^{2}-R_{\mathrm{i}}^{2}\right)}{r_{\mathrm{i}} \cos \theta_{\mathrm{i}}} \frac{d v_{\mathrm{m}}}{d t}+P_{\mathrm{e}} .
$$

Differentiation of $v_{\mathrm{o}}(t)$ is required to find $d v_{\mathrm{m}} / d t=d^{2} r_{\mathrm{m}} / d t^{2}$ and experimental noise or wall "ringing" due to confiner compressibility will yield nonphysical negative pressure values. Noise in $v_{\mathrm{o}}(t)$

$\overline{\text { reports }} d r_{\mathrm{o}} / d t$ as the wall velocity and implicitly assumes 1-D motion with $u_{\mathrm{o}}(t)=0$. This induces minimal effect on isentrope determination but a measurable effect on case velocity and position. 
can be filtered or fit with a smooth function, as has been historically done [7]. Determination of $P$ for compressible case motion is discussed in Sec. 2.4.

\subsection{Specific Volume of the Product Gas}

The specific volume $\nu_{\mathrm{p}}$ (not to be confused with radial velocity $v_{\mathrm{o}}$ ) of the product gas is determined by applying the integral forms of the mass and momentum equations inside the cylinder and across the detonation. Combination of these two equations to eliminate the downstream flow velocity $u_{\mathrm{p}}$ allows determination of $\nu_{\mathrm{p}}$ as a function of known parameters. To complete this analysis, a conical flow profile is assumed for the product gas as shown in Fig. 1.

Conservation of mass and momentum between an upstream and downstream state in the control volume of Fig. 2, in the $\hat{x}$-direction, yields

$$
\rho_{\mathrm{e}} D R_{\mathrm{i}}=2 \rho_{\mathrm{p}} \int_{0}^{r_{\mathrm{i}}} u_{\mathrm{p}} r d r
$$

and

$$
2 \rho_{\mathrm{p}} \int_{0}^{r_{\mathrm{i}}} u_{\mathrm{p}}^{2} r d r=\rho_{\mathrm{e}} D^{2} R_{\mathrm{i}}-\operatorname{Pr}_{\mathrm{i}}^{2}+F_{\mathrm{b}}+P_{\mathrm{e}} R_{\mathrm{i}}^{2}
$$

respectively. Parameter $P_{\mathrm{e}}$ is the upstream pressure, while $\rho_{\mathrm{p}}(x)$ and $u_{\mathrm{p}}(r, x)$ represent the product gas density and $\hat{x}$-component velocity, respectively. Parameter $F_{\mathrm{b}}$ is described below.

Assuming 1-D flow would simplify [6,7] derivation of $\nu_{\mathrm{p}}$ but is not consistent with this 2-D analysis. Instead, the post-detonation flow vector is assumed tangential to the tube wall at radius $r_{\mathrm{i}}$ and to the $x$-axis at the tube centerline. Flow at intermediate radii $r$ vary smoothly between these two

limits as $\theta_{\mathrm{p}}(r, x)=\arctan \left[\frac{r}{r_{\mathrm{i}}} \tan \theta_{\mathrm{i}}\right]$. Essentially, the flow at all $r$ is assumed to stream spherically outwards from a common virtual origin, whose location varies with $r_{\mathrm{i}}$ and $\theta_{\mathrm{i}}$. The total magnitude of the gas velocity $V_{\mathrm{p}}$ is assumed to only vary with $x$ and not radially, $u_{\mathrm{p}}(r, x)=V_{\mathrm{p}}(x) \cos \theta_{\mathrm{p}}(r, x)$. Thus,

$$
u_{\mathrm{p}}(r, x)=\frac{V_{\mathrm{p}}}{\left[1+r^{2}\left(\tan \theta_{\mathrm{i}} / r_{\mathrm{i}}\right)^{2}\right]^{1 / 2}}
$$

Substitution of the above relation into the mass and momentum balances allows integration and 
elimination of $V_{\mathrm{p}}$ to yield

$$
\nu_{\mathrm{p}}=r_{\mathrm{i}}^{2} A \frac{\left(\rho_{\mathrm{e}} D^{2} R_{\mathrm{i}}^{2}-P r_{\mathrm{i}}^{2}+F_{\mathrm{b}}+P_{\mathrm{e}} R_{\mathrm{i}}^{2}\right)}{\rho_{\mathrm{e}}^{2} D^{2} R_{\mathrm{i}}^{4}}
$$

where

$$
A=\left(\frac{2}{\tan \theta_{\mathrm{i}}}\right)^{2} \frac{\left(\sec \theta_{\mathrm{i}}-1\right)^{2}}{\ln \left(\sec ^{2} \theta_{\mathrm{i}}\right)}
$$

and

$$
F_{\mathrm{b}}=2 \int_{0}^{x} P(x) r_{\mathrm{i}} \tan \theta_{\mathrm{i}} d x .
$$

The terms in Eq. (8) are arranged in decreasing order of importance. The last term $P_{\mathrm{e}} R_{\mathrm{i}}^{2}$ is negligible for explosive initially experiencing atmospheric pressure. Parameter $F_{\mathrm{b}}$ is the $\hat{x}$-direction force due $P(x)$ on the tube interior, and is on the order of $1 \%$ of $\nu_{\mathrm{p}}$. Assuming 1-D product flow would reduce $A$ to unity, yielding deviations from Eq. (9) of less than $0.1 \%$ for $\theta_{\mathrm{i}}<25^{\circ}$.

\section{4 $P$ and $\nu_{p}$ for Compressible Case Motion}

Compressible case motion is often present during early expansion for detonations where $D$ exceeds the confiner sound speed. This compressibility manifests itself as ringing oscillations (e.g., $v_{\text {expt }}$ in Fig. 4). The high-impedance products drive shocks in the confiner, compressing it. When the shock reaches the free surface $r_{\mathrm{o}}$, the confiner expands outward and an expansion wave travels inward to $r_{\mathrm{i}}$, decreasing the confiner stress to $P_{\mathrm{e}}$ (neglecting any air shocks outside the case). The expansion wave reflects from $r_{\mathrm{i}}$ as a shock and the process repeats, lowering $P$ and accelerating the wall outwards with each cycle. Eventually, the decreasing $P$ can no longer shock the wall, resulting in the onset of incompressible case motion, as evidenced by the disappearance of ringing.

In reality, the process is more complicated. Detonation flow expansion in the $\hat{x}$-direction decreases $P$ independently of any radial motion. Additionally, confiner elasticity, diffraction of the radially expanding shock in the confiner, and the dispersive nature of expansion waves serve to smooth the initially discontinuous shock motion into a region of non-simple confiner flow that cannot be exactly modeled analytically.

Neglecting these non-ideal effects, the compressed case pressure can be approximated at each shock breakout from the ringing amplitude. The case will successively compress and expand along its Hugoniot and isentrope, respectively, as shown in Fig. 3. The difference between a local peak and 
minimum case velocity can thus be combined with the Hugoniot and isentrope to yield $P$ and $v_{\mathrm{i}}$ at the confiner-product interface with a shock impedance match. This $P$ can then be used to determine the degree of volumetric case compression $\nu_{\mathrm{w}} / \nu_{\mathrm{w}, 0}$. Equations (4) and (8), with $A=1$ and $F_{\mathrm{b}}=0$, can then be recalculated and combined with $P$ to specify the $1-\mathrm{D}$, compressible-case $P\left(\nu_{\mathrm{p}}\right)$ state at the shock breakout time. (One could also interpolate $P(x)$ between ringing peaks to approximate $F_{\mathrm{b}}$ for 2-D flow.) The following calculation interpolates tabulated values of $P\left(v_{\mathrm{o}}\right)$ and $\nu_{\mathrm{w}} / \nu_{\mathrm{w}, 0}(P)$ from Marsh [9] for a pure Copper confiner. The isentrope is approximated as a Hugoniot, which introduces some error $(\sim 1 \%)$, but illustrates the methodology.

\section{Application to Experiment}

Figure 4 shows the wall velocity record $v_{\text {expt }}$ (yellow) from a cylinder test experiment that was recorded with a PDV (Photon Doppler Velocimetry) probe oriented normal to the initial position of the cylinder wall. The cylinder was a 2.0-scale variant of the detonation cylinder test [2] with $R_{\mathrm{o}}=$ $61.0 \mathrm{~mm}(2.400 "), R_{\mathrm{i}}=50.8 \mathrm{~mm}(2.000 ")$ and $\rho_{\mathrm{w}}=8.94 \mathrm{~g} / \mathrm{cc}$ (Copper confiner). The cylinder was filled with PBX 9502 (95.0\% TATB, 5.0\% plastic binder) explosive at $\rho_{\mathrm{e}}=1.889 \mathrm{~g} / \mathrm{cc}$ and detonated at $D=7.653 \pm 0.002 \mathrm{~mm} / \mu \mathrm{s}$. The total tube length was $609.6 \mathrm{~mm}$ and the probe was $152.4 \mathrm{~mm}$ from the downstream (breakout) surface. A detailed description of the experiment is given elsewhere $[10]$.

Confiner compressibility effects are apparent in the form of wall ringing from $0<x / R_{\mathrm{o}}<3$. Vertical black ticks above and below the trace illustrate these local maxima and minima, respectively. The red curve is an analytic equation of the form

$$
v_{\mathrm{o}}(t)=\frac{v_{\infty}\left((t+1)^{\omega}-1\right)}{\frac{2 v_{\infty} \omega}{a_{0}}(t+1)^{\omega}-1}
$$

that was fit to the experimental trace yielding $v_{\infty}=1.563 \mathrm{~mm} / \mu \mathrm{s}, a_{0}=0.7562 \mathrm{~mm} / \mu \mathrm{s}^{2}$, and $\omega=$ 0.7382. As discussed by Hill [7], this form satisfies the kinematic constraints of the cylinder motion. Hill [7] prescribes $a_{0}$ to yield assumed values of $P_{\mathrm{CJ}}$, but this is not done in the present study as CJ behavior is not expected, given that $D$ is only $98.7 \%$ of the CJ value. Equation (11) was used with Eqs. (1) and (3) to determine $u_{\mathrm{o}}$ (blue) and $d r_{\mathrm{o}} / d t$ (green), respectively. Total wall velocity 
$V_{\mathrm{w}}=\sqrt{u_{\mathrm{o}}^{2}+v_{\mathrm{o}}^{2}}$ is also shown in black. Assuming 1-D motion will yield a velocity magnitude of $v_{\mathrm{o}}$ and $d r_{\mathrm{o}} / d t$ when using a PDV probe or streak camera, respectively, as wall velocity diagnostics. For this particular test, $d r_{\mathrm{o}} / d t$ and $v_{\mathrm{o}}$ each deviate from the actual total velocity $V_{\mathrm{w}}$ by $\pm 2.1 \%$ as as $v_{\mathrm{o}}$ approaches the terminal velocity $v_{\infty}$ and illustrate the error induced by the 1-D assumption. The differences between the wall angles $\theta_{\mathrm{o}}$ (solid curve) and $\theta_{\mathrm{i}}$ (dotted curve) are plotted against the wall angle from the 1-D analysis (dashed curve) in Fig. 5. Deviations between $\theta_{\mathrm{o}}$ and $\theta$ for 1-D are approximately $0.5^{\circ}$ at late times, while $\theta_{\mathrm{i}}$ is approximately a degree larger than $\theta_{\mathrm{o}}$ due to thinning of the cylinder wall as the radius increases.

With $v_{\mathrm{o}}, u_{\mathrm{o}}$, and $D$ known, Eqs. (2) and (3) can be integrated and parametrically plotted to yield the case shape, as shown in Fig. 1. Solid black lines represent the inner $r_{\mathrm{i}}$ and $r_{\mathrm{o}}$ outer wall contours. Dashed black lines show the initial radii $R_{\mathrm{i}}$ and $R_{\mathrm{o}}$. The center of mass $r_{\mathrm{m}}$ is shown by the dashed green line. Gray lines indicate particle trajectories of case wall elements during expansion. Blue lines are the explosive and product flow streamlines for the conical flow assumption.

The pressure and specific volume evolution with length as calculated from Eqs. (7) (solid green curve) and (8) (dotted black curve) with (9) and (10) are shown in Fig. 6. The pressure decreases inversely with distance and drops from an initial postshock value of 23.5 GPa to 3.3 GPa over a distance of $x / R_{\mathrm{o}}=1$ and a time of $4.03 \mu \mathrm{s}$. The specific volume increases at a lower rate. The dashed blue and dash-dot red curves show the error (as percent of Eq. (8)) induced in $\nu_{\mathrm{p}}$ by assuming quasi1D product flow with $\left(A=1, F_{\mathrm{b}}\right.$ as Eq. $\left.(10)\right)$ and without $\left(A=1, F_{\mathrm{b}}=0\right)$ cylinder back pressure. There is negligible difference between the 1-D and 2-D approaches, but approximating Eq. (10) as 0 results in $0.6 \%$ error in $\nu_{\mathrm{p}}$ at long times.

The green and black discrete points in Fig. 6 indicate data derived from application of the compressiblecase analysis to $v_{\text {expt }}$ (Figure 3 shows the actual impedance-matched solutions.) The compressiblecase analysis yields pressures that differ from the incompressible-case model when wall ringing is present. As the wall ringing amplitude decays, the pressures calculated from both models converge. Away from the first ring point, variations in $\nu_{\mathrm{p}}$ are less significant between the two models. As noted [6], the explosive products experience no shocks during case expansion. For prompt reaction at the detonation, the product isentrope can be visualized by parametrically plotting $P$ versus $\nu_{\mathrm{p}}$. 
Figure 7 shows both the experimentally measured isentrope derived from the present incompressiblecase analysis (black curve) and the hydrocode-calibrated JWL isentrope (red) for PBX 9502 at the CJ detonation state [5]. ${ }^{4}$ As with the 1-D approach [7], the experimental incompressible isentrope is lower than the JWL isentrope by about $30 \%$ over $1<P<15$ GPa.

Applying the compressible approach (Sec. 2.4) over $0<x / R_{\mathrm{o}}<3$ yields the green points, which are seen to agree well with the JWL. The fifth and sixth points are slightly above the JWL. This discrepancy may be due to confiner elasticity becoming significant near the transition from compressible-to-incompressible case motion. The green, dashed line blends the compressible- (for $\left.0<x / R_{\mathrm{o}}<2\right)$ and incompressible-case (for $\left.x / R_{\mathrm{o}} \geq 2\right) P\left(\nu_{\mathrm{p}}\right)$ values.

This analysis demonstrates that neglecting case compressibility results in decreased inferred $P$ values, but that impedance matching the case ringing very closely approximates the reference JWL isentrope. The slightly lower compressible-case values relative to the JWL may be due to the shock diffraction effects discussed in Sec. 2.4 or because the experimental value of $D$ is sub-CJ and thus releases slightly less energy $\left(e=-\int P d \nu_{\mathrm{p}}\right)$. Hydrocode errors in the JWL determination may also be present, but are not considered.

The largest pressure value derived from the initial compressible cylinder motion (green point at $\sim 16$ $\mathrm{GPa})$ is from the shock breakout near $x / R_{\mathrm{o}}=0$. As this is the first measured motion, no direct isentrope measurement is possible above this value for the cylinder test. All methods, including the hydrocode calibration, must extrapolate to obtain higher $P\left(\nu_{\mathrm{p}}\right)$ predictions. In the present study, an exponential fit to the dashed green data (Fig. 7) is used to extrapolate the compressible-case isentrope pressures above $16 \mathrm{GPa}$. The computed isentrope curves can be fit to the analytic JWL form or interpolated to serve as a look-up table.

\subsection{Estimating the Post-Detonation State}

Conservation of mass and momentum specify that the Rayleigh line must be coincident with the product isentrope at the post-detonation state, immediately downstream of the detonation. In order

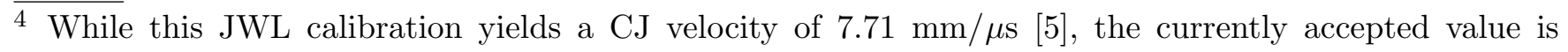
between $7.75-7.80 \mathrm{~mm} / \mu \mathrm{s}$, indicating that the CJ reference curve may underpredict the actual CJ energy. 
for the detonation to be stable, the Rayleigh line and isentrope must also be tangent at this point. Extrapolations above the first directly measured $\left(\nu_{\mathrm{p}}, P\right)$ point will need to take these constraints into account. The Rayleigh line is $P_{\mathrm{r}}=D^{2}\left(\nu_{\mathrm{e}}-\nu\right) / \nu_{\mathrm{e}}^{2}$ where $\nu_{\mathrm{e}}=1 / \rho_{\mathrm{e}}$ and $\nu$ is evaluated immediately post-detonation. The Rayleigh line is plotted, using $D$ from the cylinder test, as the blue line on Fig. 7. The intersection points are shown by " $\times$ " symbols on Fig. 7 for the compressible-case (green) and incompressible-case (black) isentropes. The CJ point from the JWL (red) reference curve is also shown. As the CJ velocity differs from the experimental $D$, the JWL CJ value is not intersected by the experimental Rayleigh line. The specific values are:

$$
\begin{array}{r}
\text { CJ: }\left(\nu_{\mathrm{p}}, P\right)_{\mathrm{CJ}}=(0.382 \mathrm{cc} / \mathrm{g}, 30.2 \mathrm{GPa}) \\
\text { Comp. wall: }\left(\nu_{\mathrm{p}}, P\right)_{\mathrm{c}}=(0.397 \mathrm{cc} / \mathrm{g}, 27.6 \mathrm{GPa}) \\
\text { Incomp. wall: }\left(\nu_{\mathrm{p}}, P\right)_{\mathrm{ic}}=(0.403 \mathrm{cc} / \mathrm{g}, 26.4 \mathrm{GPa})
\end{array}
$$

Both $P_{\mathrm{c}}$ and $P_{\text {ic }}$ are less than the CJ prediction, however $P_{\mathrm{c}}$ may be a more accurate extrapolation given that the compressible-case isentrope agrees more closely with the CJ isentrope. The heat of detonation can be calculated as the difference between the areas under the Rayleigh line and isentrope curves.

\subsection{Inverting the Method}

With a specified isentrope $P\left(\nu_{\mathrm{p}}\right), D$ and post-detonation state, it is also possible to reverse the above process to solve the resulting cylinder expansion with the incompressible-case model. This involves solving a coupled system of ordinary differential equations involving $d r_{\mathrm{o}} / d t$ from Eq. (3), $d v_{\mathrm{o}} / d t$ from Eq. (7), and $d \nu_{\mathrm{p}} / d t$ from Eq. (8). Assuming 1-D motion allows determination of $P\left(r_{\mathrm{o}}\right)$ directly from $P\left(\nu_{\mathrm{p}}\right)$. Thus, only $d r_{\mathrm{o}} / d t$ from Eq. (3) and $d v_{\mathrm{o}} / d t$ from Eq. (7) need to be integrated together. Either approach can be used to predict fragment velocities.

\section{Conclusions}

A two-dimensional, analytic model has been developed to determine the cylinder motion and detonation product isentrope from experimental measurements of cylinder tests. Prediction of the outer 
case motion is always exact, while the inner case motion and product isentrope predictions are exact for incompressible wall motion and a conical product flow profile. For compressible wall motion, an approximate, analytic, impedance-matching model was also presented.

This analytic methodology was applied to experimental PBX 9502 cylinder expansion data with compressible wall motion. The measured isentrope using the impedance-matching model agreed well with the reference value established from direct numerical simulation of experiment, while the isentrope from the incompressible-wall model underpredicted pressure over a large range of product densities.

\section{Acknowledgements}

This work was supported by the U.S. Department of Energy via Campaign 2, "Dynamic Material Properties" and other programs.

\section{References}

[1] J. Kury, H. Hornig, E. Lee, J. McDonnel, D. Ornellas, M. Finger, F. Strange, M. Wilkins, in: Proc. 4th Intl. Det. Symp., Naval Ordnance Laboratory, ACR-126, pp. 3-13.

[2] T. Gibbs, A. Popolato, in: LASL Explosive Property Data, University of California Press, 1980, pp. 234-249.

[3] P. Souers, J. Forbes, L. Fired, W. Howard, S. Anderson, S. Dawson, P. Vitello, R. Garza, Propellants, Explosives, Pyrotechnics 26 (2001) 180-190.

[4] P. Souers, L. Lauderbach, R. Garza, J. L. Ferranti, P. Vitello, Propellants, Explosives, Pyrotechnics 38 (2013) 419-424.

[5] B. Dobratz, P. Crawford, in: LLNL Explosives Handbook. Properties of Chemical Explosives and Explosive Simulants, University of California, 1985, pp. 8-21 to 8-23.

[6] G. Taylor, Mechanics of Fluids, Scientific Papers of GI Taylor 2 (1941) 277-286.

[7] L. Hill, in: Proceedings of the 21st ISSW, pp. 1-6. Paper 2780.

[8] L. Davis, L. Hill, in: AIP Conference Proceedings, volume 620, p. 165. 
[9] S. Marsh, in: LASL Shock Hungoniot Data, University of California, 1980, pp. 57-60.

[10] S. Jackson, in: Proc. 15th Intl. Det. Symp., Office of Naval Research, To Appear in 2014. 


\section{Figures}

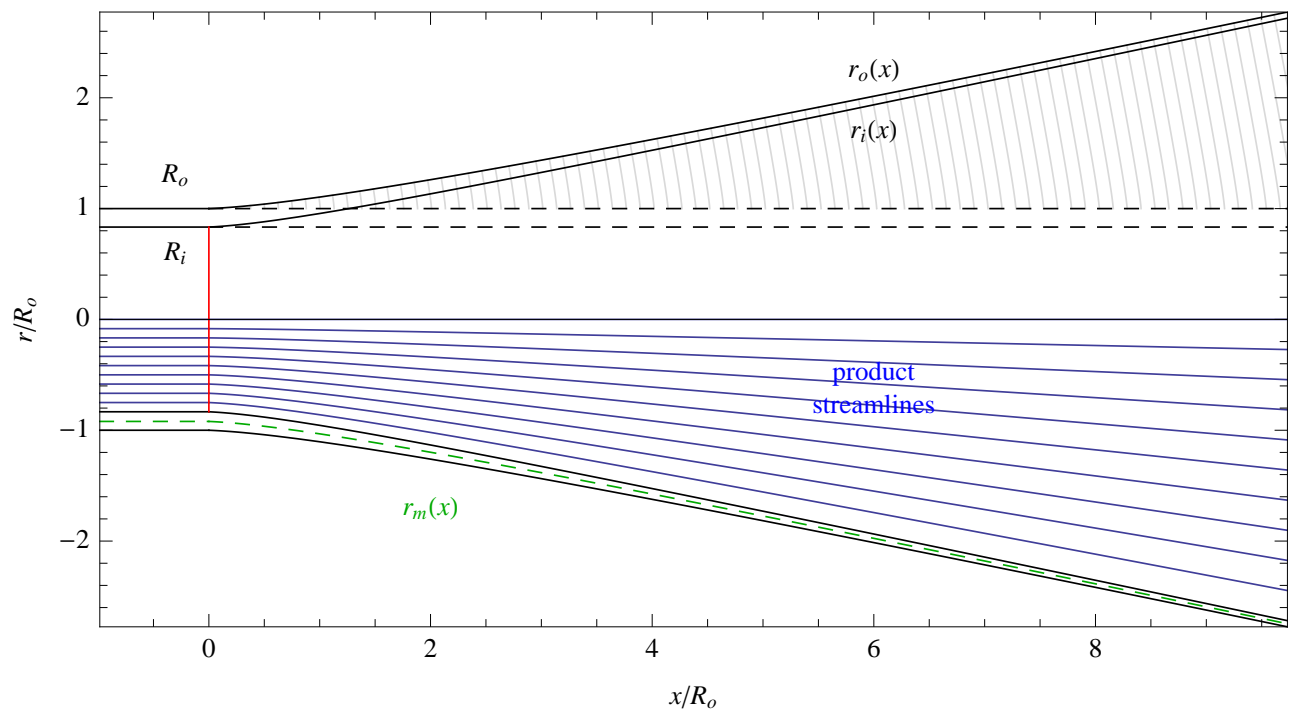

Fig. 1. Cylinder test geometry. Detonation location is the red vertical line. Solid black lines are case inner $r_{\mathrm{i}}$ and outer $r_{\mathrm{o}}$ walls, with initial positions $R_{\mathrm{i}}$ and $R_{\mathrm{o}}$ shown as dashed black lines. Green, dashed line is the case center of mass $r_{\mathrm{m}}$. Gray lines denote particle paths of the case wall elements during expansion. Blue lines are explosive/product flow streamlines.

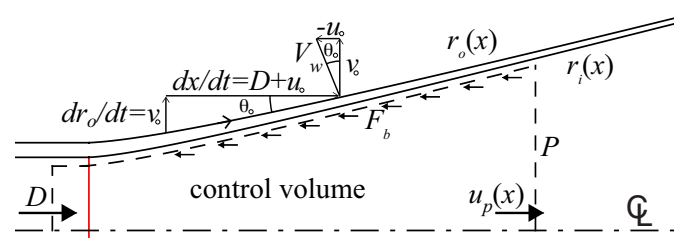

Fig. 2. Trigonometric relations for $\theta_{\mathrm{o}}$ and the control volume (dashed line) for determination of $\nu_{\mathrm{p}}$.

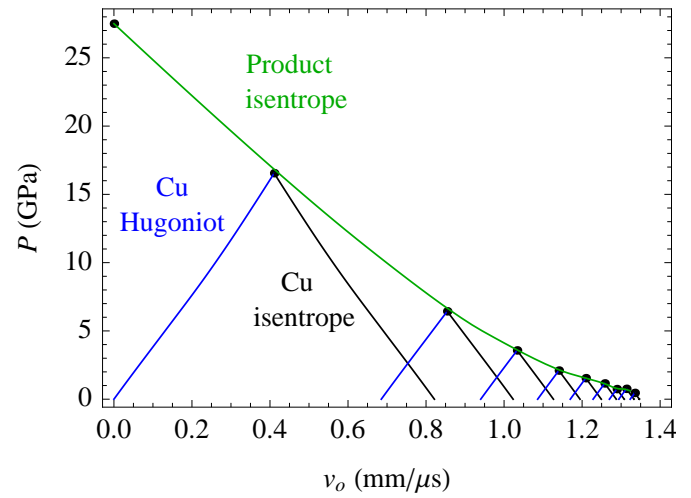

Fig. 3. Confiner and product expansion in the $P-v_{\mathrm{o}}$ plane via impedance matching. 


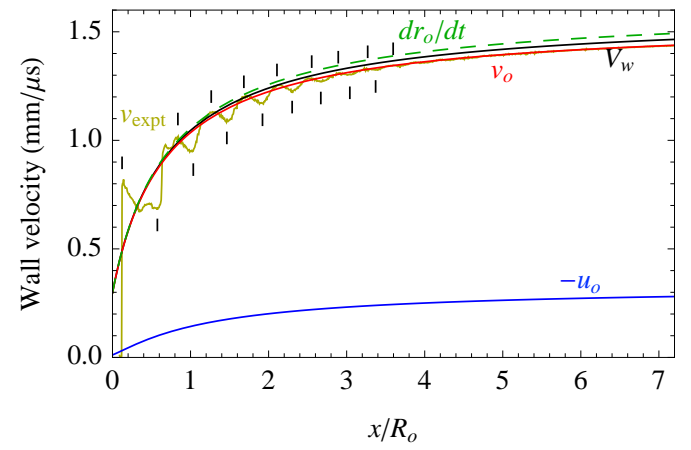

Fig. 4. Velocity-position profiles.

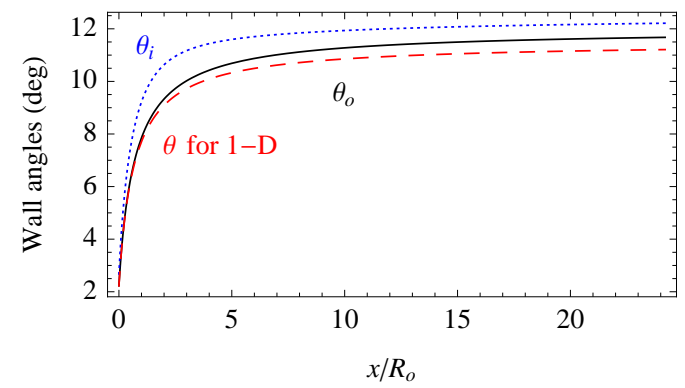

Fig. 5. Wall angles.

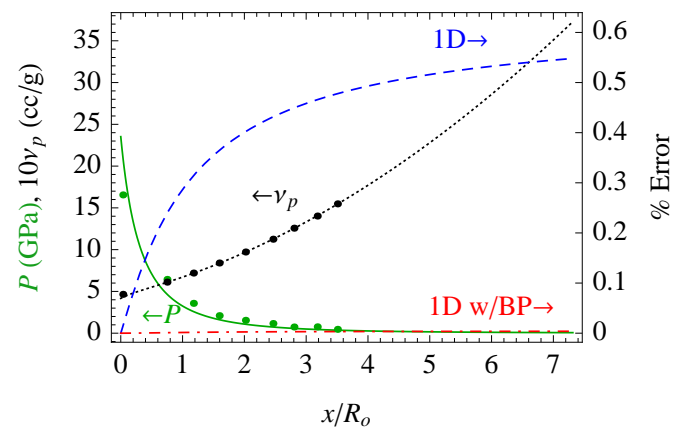

Fig. 6. Pressure, $\nu_{\mathrm{p}}$ and $\nu_{\mathrm{p}}-$ model error.

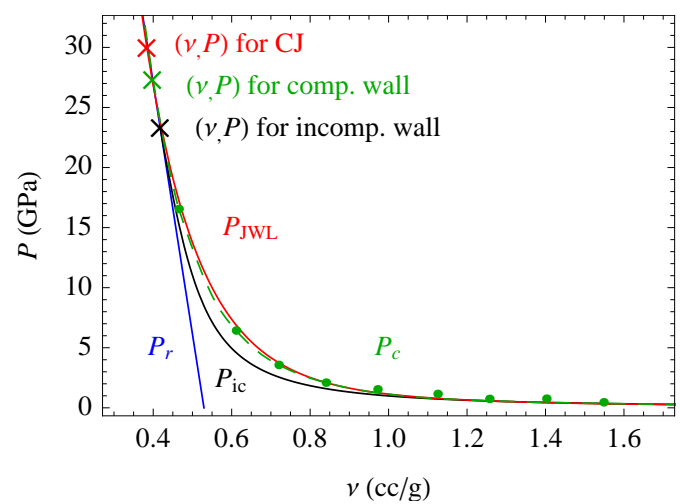

Fig. 7. CJ JWL (red), experimental incompressible-case (black) and compressible-case (green) isentropes. Peak post-detonation states are marked with " $\times$ " symbols. 


\section{Figure Captions}

Fig. 1: Cylinder test geometry. Detonation location is the red vertical line. Solid black lines are case inner $r_{\mathrm{i}}$ and outer $r_{\mathrm{o}}$ walls, with initial positions $R_{\mathrm{i}}$ and $R_{\mathrm{o}}$ shown as dashed black lines. Green, dashed line is the case center of mass $r_{\mathrm{m}}$. Gray lines denote particle paths of the case wall elements during expansion. Blue lines are explosive/product flow streamlines.

Fig. 2: Trigonometric relations for $\theta_{\mathrm{o}}$ and the control volume (dashed line) for determination of $\nu_{\mathrm{p}}$.

Fig. 3: Confiner and product expansion in the $P-v_{\mathrm{o}}$ plane via impedance matching.

Fig. 4: Velocity-position profiles.

Fig. 5: Wall angles.

Fig. 6: Pressure, $\nu_{\mathrm{p}}$ and $\nu_{\mathrm{p}}-$ model error.

Fig. 7: CJ JWL (red), experimental incompressible-case (black) and compressible-case (green) isentropes. Post-detonation states are marked with " $\times$ " symbols. 